\title{
Mailuoning and Luteolin Prevents High Glucose-induced Human Retinal Microvascular Endothelial Cell Apoptosis in Diabetic Retinopathy
}

\begin{abstract}
Y. YANG, H. Z. LIU1', P. C. HU², J. YANG ${ }^{2}$, T. X CAI ${ }^{2}$, F. K ZHU, P. F LI, T. JIANG ${ }^{1}$, P. CAO² AND W. WANG*
Department of Ophthalmology, The Second Affiliated Hospital of Nanjing University of Chinese Medicine, No. 23 Nanhu Road, Nanjing, Jiangsu 210000, 'Department of Ophthalmology, Nanjing Central Hospital, 116 Chengxian Street, Nanjing, Jiangsu 210000, 2Department of Ophthalmology, Affiliated Hospital of Nanjing University of Chinese Medicine, No. 155, Hanzhong Road, Nanjing, Jiangsu 210000, China
\end{abstract}

Yan et al.: Mailuoning and luteolin prevents retinal microvascular endothelial cell apoptosis

\begin{abstract}
The present study is aimed to determine the mechanism of the protective effect of mailuoning and its main component luteolin on diabetic retinopathy. Human retinal microvascular endothelial cells were obtained and cultured in high glucose medium. Different concentrations of mailuoning and luteolin were added to the cells. MTT, flow cytometry and TUNEL staining assays were performed to investigate the effects of mailuoning on high glucose-induced proliferation and apoptosis in human retinal microvascular endothelial cells. Enzyme-linked immunosorbent assay was used to detect changes in inflammatory factors, and qPCR was used to detect the expression of apoptosis-related protein Bax and antiapoptotic protein Bcl2. Western blot was used to detect changes of VEGFR2 and its phosphorylated p-VEGFR2. After adding mailuoning, cell viability of human retinal microvascular endothelial cells was significantly decreased in a concentration-dependent manner while $200 \mu \mathrm{g} / \mathrm{ml}$ mailuoning was the highest concentration without cytotoxic effects. Under high glucose conditions, apoptosis of human retinal microvascular endothelial cells was remarkably increased in a concentration-dependent manner and $100 \mu \mathrm{g} / \mathrm{ml}$ mailuoning was the optimum concentration on human retinal microvascular endothelial cells. Further studies indicated that mailuoning and luteolin inhibited the expression of apoptotic protein Bax and upregulated the expression of antiapoptotic protein Bcl-2. At the same time, inhibited the expression of inflammatory factors IL-6, TNF $\alpha$ and IL-1ß. Mechanism studies have shown that mailuoning and luteolin could inhibit p-VEGFR2. Mailuoning and luteolin could prevent high glucose-induced human retinal microvascular endothelial cell apoptosis in diabetic retinopathy, suggesting that mailuoning and luteolin could be useful in the clinical treatment of diabetic retinopathyn.
\end{abstract}

Key words: Mailuoning, luteolin, p-VEGFR2, diabetic retinopathy, RMECs

Diabetic retinopathy (DR), a type of visual impairment and blindness-producing complications of diabetes mellitus occurring among working-aged adults around the world ${ }^{[1]}$. Several DR patients may have accompanying serious complications, such as diabetic heart disease, high blood pressure and kidney diseases. Medical treatment include laser treatment and adjuvant oral medication to control deterioration of fundus lesions and to prevent retinal bleeding ${ }^{[2-4]}$. However, traditional therapy may cause certain poor outcomes and side effects. Traditional Chinese medicine has accumulated abundant information revealing the etiology, pathogenesis and treatment of DR. Although recent studies have reported the pathogenesis of retinal microvascular endothelial cells (RMECs) apoptosis, the underlying mechanisms were largely unknown ${ }^{[5,6]}$.

DR histopathologically manifested as retinal blood vessels weakness and endothelial cell death, only with bare basement membrane of vessel wall, vascular degradation leading to retinal tissue ischemia, reduced oxygen and nutrition supply, blood-retinal barrier damage causing release of many proinflammatory factors, which stimulate residual vascular leakage and pathological formation of new blood vessels. Repair of damaged vascular tissue and reconstruction is the key to prevent $\mathrm{DR}$ from developing into a proliferative type.

*Address for correspondence

E-mail: libu7300369269925@163.com

Special Issue 5, 2020 
Mailuoning is a traditional formula, mainly composed by Radix Scrophulariae, Dendrobium, Achyranthes bidentata, honeysuckle and Radix codonopsis ${ }^{[7]}$. A body of evidence reported that mailuoning could be used to treat thromboangiitis obliterans, arterial occlusion and acute ischemic stroke. Moreover, mailuoning injection was used for improving microcirculation, blood flow and cardiovascular diseases ${ }^{[8,9]}$. However, the effect of mailuoning on DR remained largely unknown. The dried flower buds of the first blooming flowers of honeysuckle, Lonicera japonica Thunb. are the main components of mailuoning ${ }^{[10-12]}$. It has the effect of clearing away heat and detoxification, cooling wind and heat ${ }^{[13-15]}$. It is widely used as antiinflammatory in the clinic. Chemical analysis showed that honeysuckle mainly contains luteolin ${ }^{[16]}$. In the present study, the effects of mailuoning and luteolin on RMEC apoptosis was evaluated in order to assess the promising role of mailuoning and luteolin in the clinical treatment of DR.

\section{MATERIALS AND METHODS}

Mailuoning injection, provided by Nanjing Jinling Pharmaceutical Company Ltd., is a compound preparation made by chemical extraction of Radix scrophulariae, Dendrobium, Achyranthes bidentata, honeysuckle and Radix codonopsis. Luteolin was purchased from Dalian Meilun Biotechnology Co., Ltd.

\section{Cell culture:}

Human RMECs (MZ-2935) were obtained from Mingzhou Biotechnology (Ningbo, China) and cultured in Dulbecco's modified Eagle's medium (DMEM; Gibco; ThermoFisher Scientific, USA) supplemented with $10 \%$ fetal bovine serum and $5 \%$ penicillinstreptomycin (Invitrogen; ThermoFisher Scientific, USA).

To explore damage to RMECs in DR, high glucose conditions were simulated. Briefly, cells were exposed to normal glucose $(5 \mathrm{nM})$ and high glucose $(30 \mathrm{nM})$ and cultured for $24 \mathrm{~h}$ at $37^{\circ}$ under $5 \% \mathrm{CO}_{2}$ in a humidified atmosphere. Culture medium was changed every $24 \mathrm{~h}$ before performing experiments.

\section{Cell viability assay:}

Cells were seeded in 96-well plates at a density of $1 \times 105$ cells/well and starved for $12 \mathrm{~h}$. Then, different concentrations of mailuoning (50, 100, 200, 250, 300 $\mu \mathrm{g} / \mathrm{ml}$ ) were added and cultured for another $24 \mathrm{~h}$. Cell viability was measured using the MTT assay under the manufacturer instructions. First, cells were incubated with $0.8 \mathrm{mg} / \mathrm{ml} \mathrm{MTT}$ for $4 \mathrm{~h}$, washed thrice with
PBS, DMSO was added to complete dissolution. The absorbance at $450 \mathrm{~nm}$ was measured on a Microplate Reader (Bio-Tek Technologies, USA).

\section{TUNEL staining assay:}

TUNEL staining assay was applied to determine apoptotic cells following the manufacturer's protocol. In brief, the collected cells were treated with low and high glucose and $100 \mu \mathrm{g} / \mathrm{ml}$ mailuoning. Then, percent apoptotic cells was detected by terminal deoxyribonucleotidyl transferase-mediated dUTPbiotin nick end labelling method. Afterward, the nuclei were labelled with 4',6-diamidino-2-phenylindole (DAPI). Finally, positive cells were imaged under a fluorescent microscope and the ratio was calculated by TUNEL-positive cells to the total cells.

\section{Flow cytometry assay:}

Cells were seeded into 96-well plates at the density of $1 \times 105$ cells/well and cultured at $5 \% \mathrm{CO}_{2}$ in a humidified atmosphere at room temperature. The flow cytometry analysis was performed using Annexin V-FITC-PI Apoptosis Detection Kit (Vazyme Biotech, Nanjing, China). In brief, cells were washed with PBS and collected via centrifugation at $1000 \mathrm{rpm}$ for 5 min. Then, the treated cells were stained with Annexin V/PI at room temperature for $15 \mathrm{~min}$. The data were assessed by flow cytometry (FACSCalibur; Bio-Rad Laboratories, Inc., Hercules, CA, USA) and analyzed using BD Accuri C6.

\section{RT-qPCR:}

For real-time fluorescence quantitative PCR, TRIzol (Invitrogen, USA), TriPure Isolation Reagent kit (Roche, USA) and Gene JETRNA purification kit (Invitrogen) were used to collect cellular RNA after different treatments. Reaction conditions were as follows, random primers and cDNA was mixed and incubated at $70^{\circ}$ for $5 \mathrm{~min}$, quickly cooled on ice for $5 \mathrm{~min}$, buffer, reverse transcriptase, nucleotides and magnesium ions were added, annealed at $25^{\circ}$ for $15 \mathrm{~min}$ and extended at $42^{\circ}$ for $60 \mathrm{~min}$. Reaction conditions maintained were, pre-denaturation at 95 for $5 \mathrm{~min}$, denaturation at $95^{\circ}$ for $1 \mathrm{~min}$, annealing at $60^{\circ}$ for $30 \mathrm{~s}$, and extension at $70^{\circ}$ for $30 \mathrm{~s}$, for a total of 45 cycles. KAPASYBRFASTqPCRMasterMix (KapaBiosystems, USA) and CFX96 (waveguide, USA) RT-qPCR was used to detect the amplified fluorescence intensity and the 2- $\Delta \Delta \mathrm{CT}$ method was used to calculate gene expression level. The primers used in this study were, $\beta$-actin, F- 5'-CCTGTACGCCAACACAGTGC-3', R - 5 ' - AT A C T C C T G C T T G C T G AT C C - 3' ; 
the primer sequences of Bcl-2, F- proliferation (fig. 1A). TUNEL staining showed A T G C C T T T G T G G A A C T A T A T G G C, R- $\quad$ GGTATGCACCCAGAGTGATGC; Bax, F- TGAAGACAGGGGCCTTTTTG, R-AATTCGCCGGAGACACTCG.

\section{Western blot:}

Each group of cells was lysed with RIPA lysate. After homogenization, the protein was extracted. A small amount of supernatant was aspirated and the protein was quantified by the BCA method. Afterwards, the remaining supernatant was transferred to a new $1.5 \mathrm{ml}$ centrifuge tube and the corresponding volume of $5 \mathrm{ml}$ was added, loading buffer, boiled in boiling water for $10 \mathrm{~min}$. Equipped with glue and loaded after centrifugation. Protein electrophoresis was performed using polyacrylamide gel, $90 \mathrm{~mA}$ constant flow membrane, $50 \mathrm{~g} / 1$ milk blocking for $1 \mathrm{~h}$, and rabbit antiVEGFR2 and p-VEGFR2 primary antibodies (Abcam, 1:1000) were incubated overnight. On the second day, the corresponding secondary antibody (1:10000) labelled with horseradish peroxidase (HRP) was incubated and developed with ECL. The Bio-Rad digital imaging system was used to take pictures and save the analysis pictures. Finally, further statistical analysis.

\section{Detection of inflammatory factors:}

The cells were collected after different treatments and the corresponding ELISA kit was used for detection according to the instructions of the kit.

\section{Statistical analysis:}

All the statistical data were presented as mean \pm standard deviation (SD). SPSS 17.0 for windows (Chicago, IL, USA) software and GraphPad Prism 5.0 (GraphPad Software, Inc., La Jolla, CA, USA) were used to analyze the data. Student's t-test was applied to assess the differences between the two groups while one-way analysis of variance (ANOVA) followed by Dunn's post hoc test was performed to distinguish differences among 3 or more groups. Values of $p<0.05$ suggested statistical significance. All the experiments were performed in triplicate.

\section{RESULTS AND DISCUSSION}

In order to study the protective effect of mailuoning on RMECs, a high glucose-induced diabetic retinopathy cell model was constructed. Cell proliferation and apoptosis were detected by high concentration of glucose (5 and $30 \mathrm{nM}$ ). The experimental results showed that high glucose induced inhibition of cell that high glucose-induced RMEC apoptosis, and the apoptosis rate gradually increased with the increase of glucose concentration (fig. 1B). Flow cytometry detection results were consistent with TUNEL detection results (fig. 1C). Further studies showed that high glucose induction could up-regulate the expression of Bax (fig. 1D) and inhibit the expression of antiapoptotic protein Bcl-2 (fig. 1E). The results indicated that high glucose could inhibit RMEC proliferation and promote apoptosis. Further results of cellular inflammatory cytokines showed that the expression levels of IL-6, TNF $\alpha$, LDH, IL- $1 \beta$ and MCP- 1 could be upregulated by high glucose concentration, which was dose-dependent, and the release of inflammatory cytokines increased with the increase of glucose concentration (fig. 2).

In order to determine the protective concentration range of mailuoning on RMECs without cytotoxic effects, the cells were treated with mailuoning at different concentrations. The results showed that mailuoning had no significant inhibition on RMECs within the range of $200 \mu \mathrm{g} / \mathrm{ml}$ (fig. 3A). Fig. 3B showed that the cell proliferation capacity was significantly reduced after high glucose treatment compared to the control group $(\mathrm{p}<0.05)$. However, it was found that the ability of cells to proliferate recovered after treatment with mailuoning in a concentration-dependent manner. TUNEL staining results showed the protective effect of mailuoning on high glucose-induced apoptosis of RMECs (fig. 3C and 3D). The results showed that the apoptosis of RMECs cells increased after high glucose treatment and the apoptosis rate decreased significantly after adding mailuoning. Flow cytometry results were consistent with TUNEL results. As shown in fig. 3E, the apoptosis rate was significantly increased after high glucose treatment, which significantly decreased after the addition of mailuoning. The above experimental results suggested that mailuoning inhibited high glucose-induced apoptosis.

In order to further study the mechanism of mailuoning treatment on DR, the signal pathway was studied after mailuoning treatment. The detection results of apoptotic proteins showed that the expression of Bax was upregulated by high glucose, while the expression of Bax was inhibited by treatment with mailuoning in a dose-dependent manner (fig. 4A). The results of Bcl2 expression showed that the expression of $\mathrm{Bcl}-2$ was inhibited by high glucose, while the expression of Bcl-2 was upregulated by mailuoning (fig. 4B). Western blot results demonstrated high glucose-induced upregulation 
A

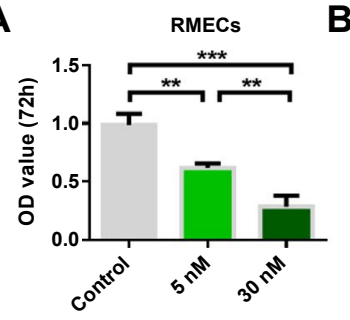

B
Control

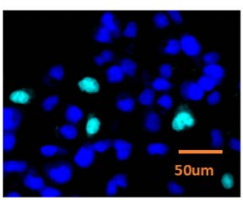

$5 \mathrm{nM}$

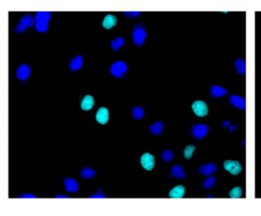

$30 \mathrm{nM}$

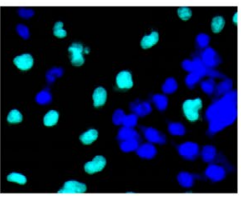

C

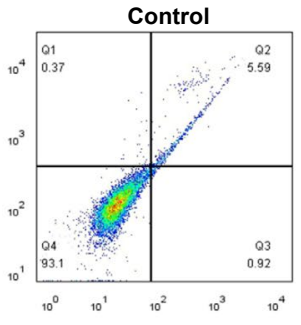

D

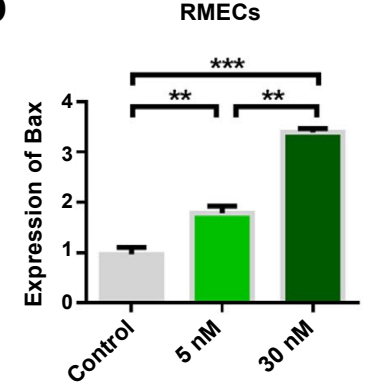

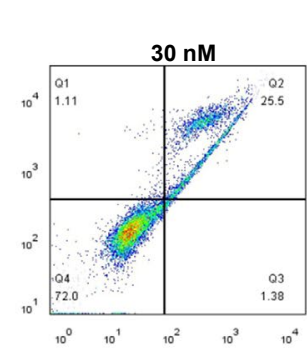

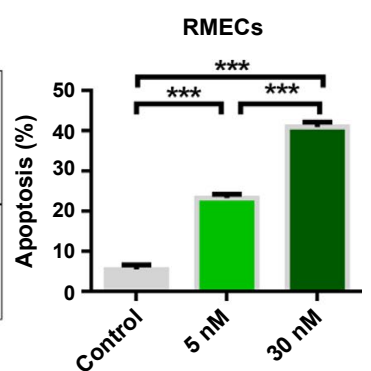

E

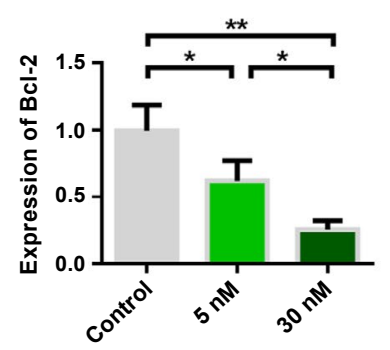

Fig. 1: Effect of high glucose on proliferation, apoptosis and apoptotic proteins of RMECs A. High glucose inhibited proliferation of RMECs; B. TUNEL detection of high glucose-induced RMECs apoptosis; C. Flow cytometry detection of high glucose-induced apoptosis of RMECs; D. Bax detection; E. Bcl-2 detection in retinal microvascular endothelial cells (RMECs)

A

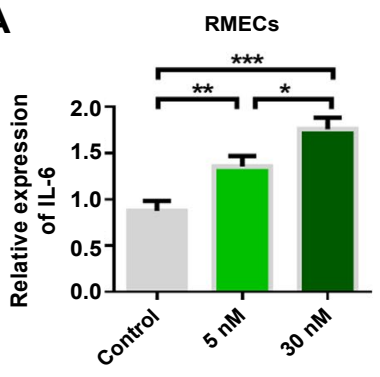

D

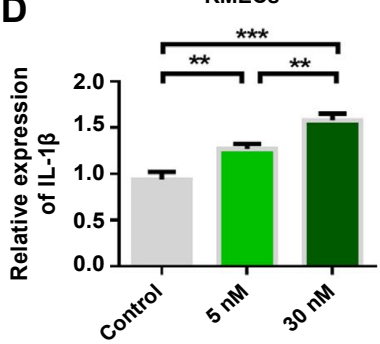

B

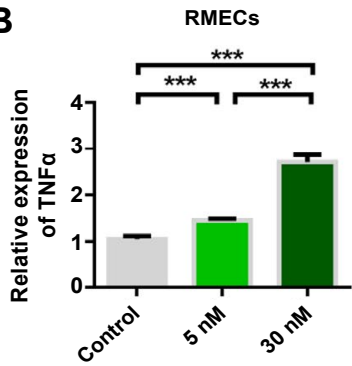

E

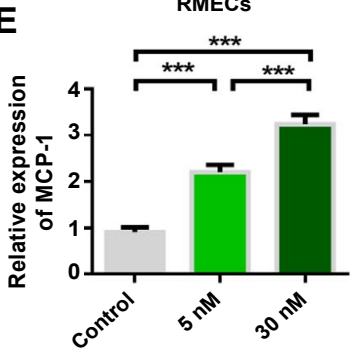

C RMECs

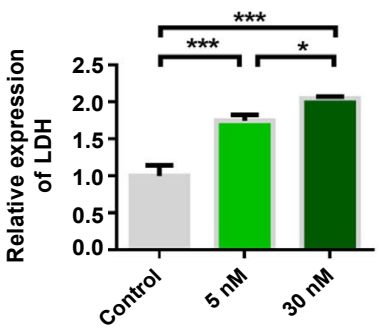



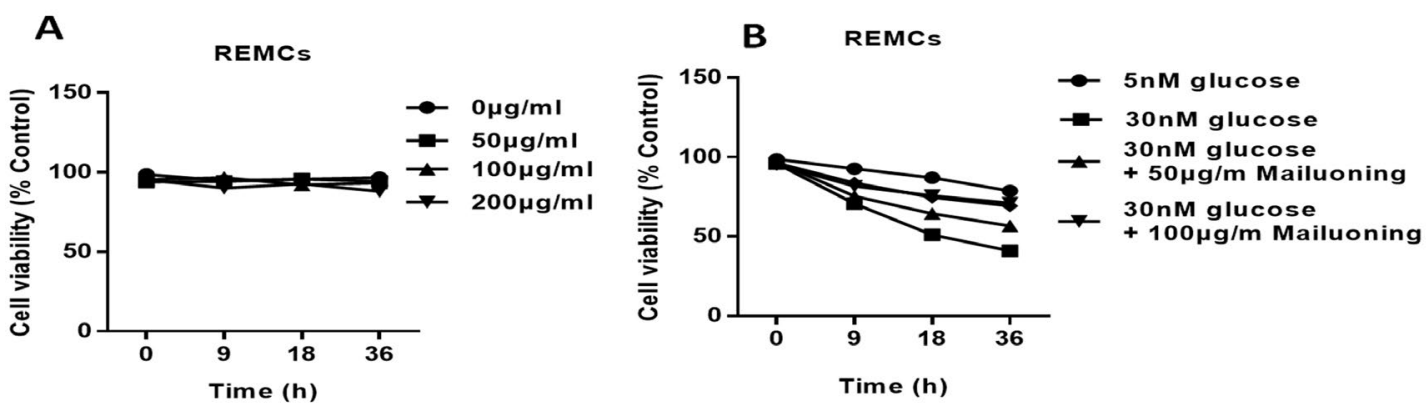

C
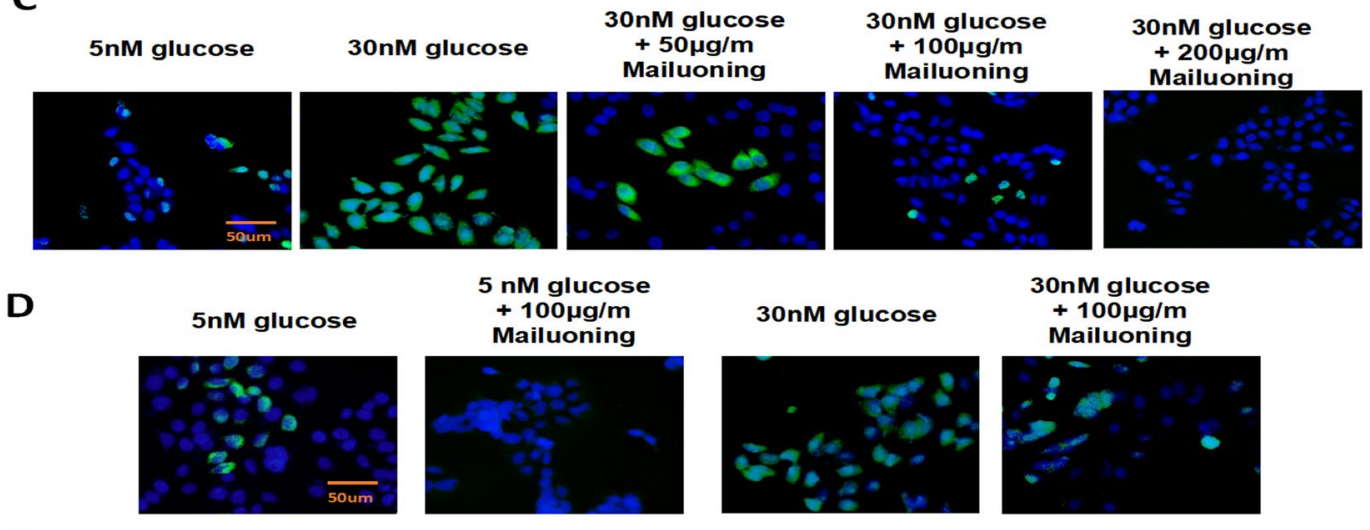

30n M glucose

$\mathbf{E}$

$5 n M$ glucose
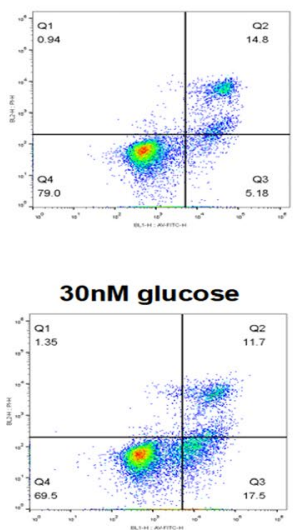

$5 \mathrm{nM}$ glucose $+100 \mu \mathrm{g} / \mathrm{m}$

Mailuoning

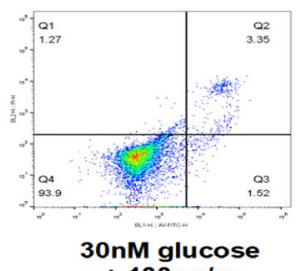

$30 \mathrm{nM}$ glucos
$+100 \mu \mathrm{g} / \mathrm{m}$

Mailuoning

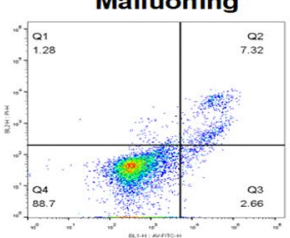
$+100 \mu g / m$
Mailuoning

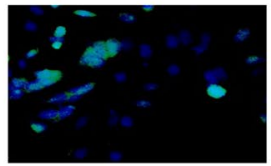

RMECs

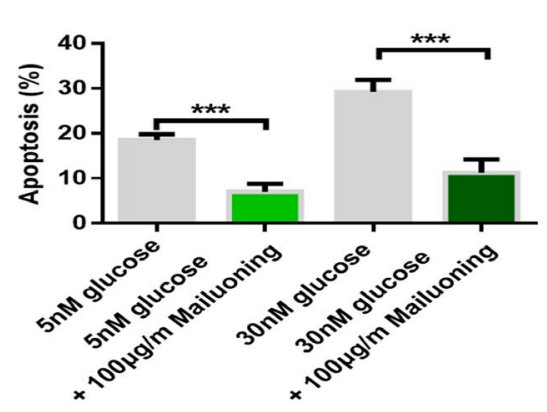

Fig. 3: Protective effect of mailuoning on RMECs

Effect of different concentrations of mailuoning on A. RMECs viability. RMECs were cultured with different concentrations of mailuoning for 9,18 and $36 \mathrm{~h}$ followed by detection of cell viability with MTT assay, B. high glucose-

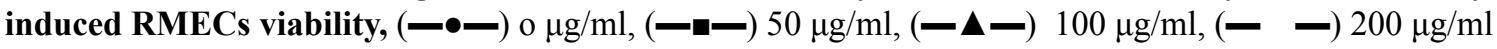

C. high glucose-induced RMECs apoptosis. RMECs were cultured with different concentrations of mailuoning under high glucose conditions for $48 \mathrm{~h}$ and cell viability was measured using TUNEL staining and (-•-) $5 \mathrm{nM}$ glucose

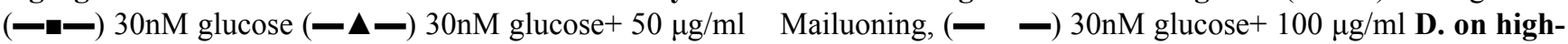
glucose-induced RMECs apoptosis TUNEL staining and E. flow cytometry assay in retinal microvascular endothelial cells (RMECs)

of p-VEGFR2 expression, while mailuoning inhibited the expression of p-VEGFR2 (fig. 4C). The detection results of cellular inflammatory factors showed that the contents of IL-6 and MCP-1 were up-regulated by high glucose, while the expression levels of IL-6 and MCP1 were inhibited by mailuoning (fig. 4D and 4E). The results showed that mailuoning inhibited p-VEGFR2.
Fig. 5A shows the structure of luteolin. The results of cell proliferation assay showed that high glucose induced inhibition of RMECs proliferation, while luteolin restored RMECs proliferation (fig. 5B). The detection results of apoptotic proteins showed that the expression of Bax was up-regulated by high glucose, while the expression of Bax was inhibited by luteolin 
A
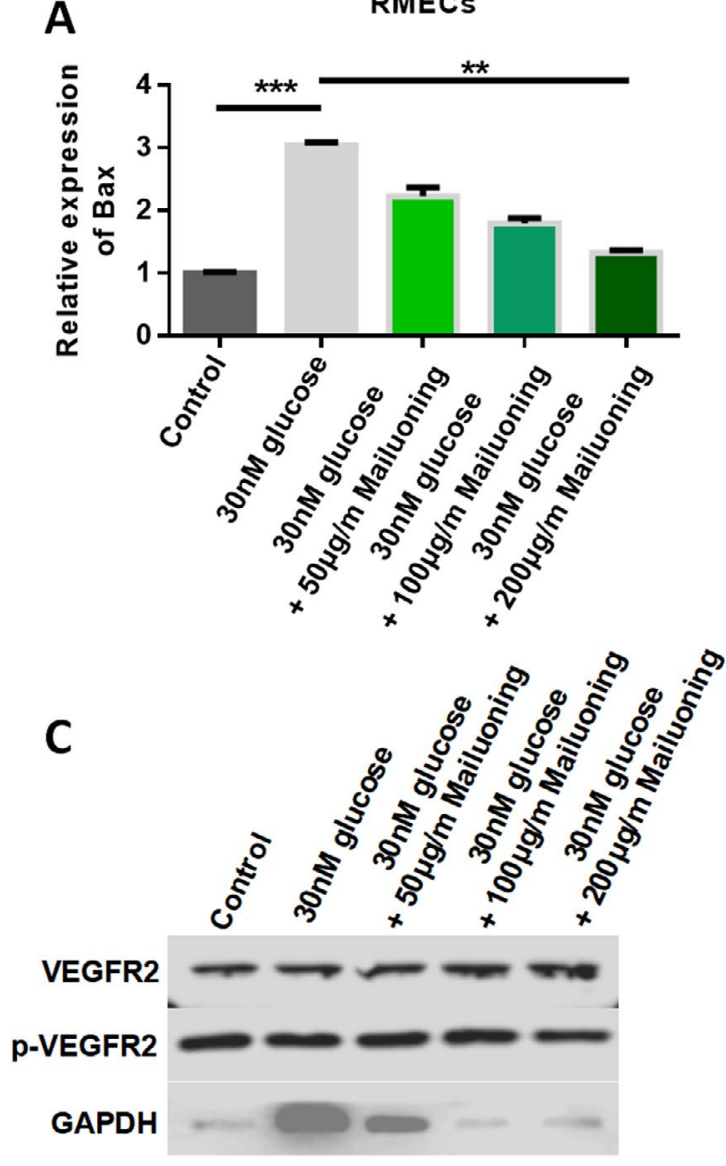

$\mathbf{E}$

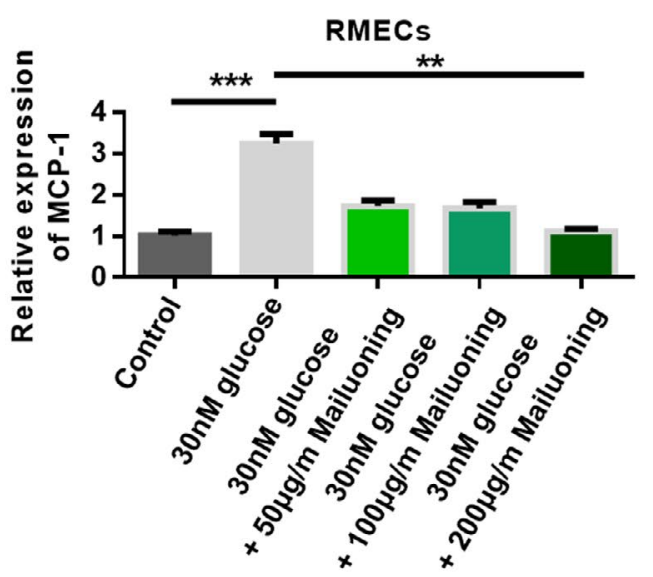

B

RMECS

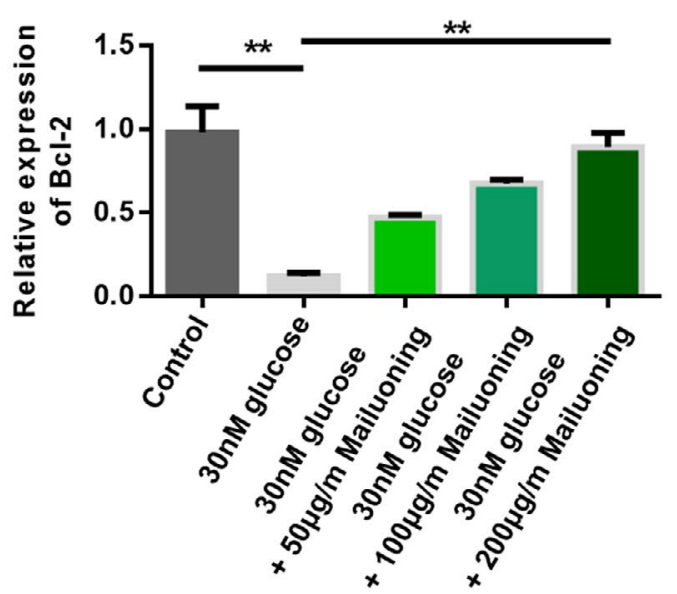

D

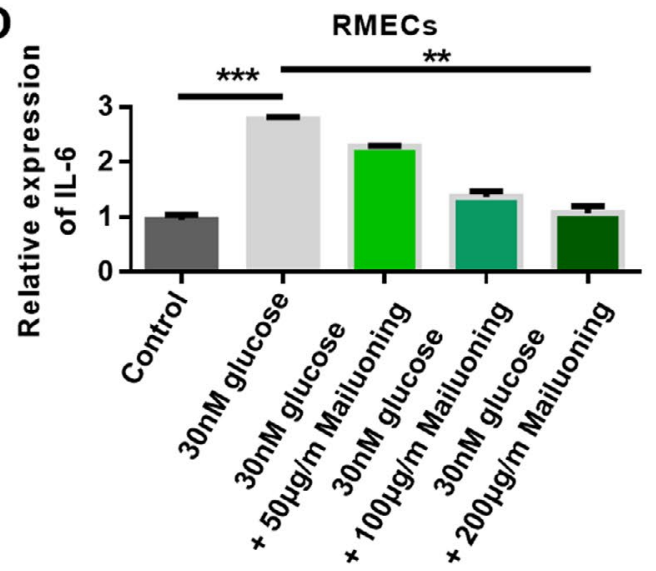

Fig. 4: mailuoning treatment on high glucose-induced effect on inflammatory factors in RMECs

Mailuoning treatment reversed the high glucose-induced effects on A. Bax, B. Bcl-2, C. VEGFR2 and p-VEGFR2, D. IL-6 and E. MCP-1 in retinal microvascular endothelial cells (RMECs)

treatment (fig. 5C). The results of the detection of Bcl-2 expression showed that the expression of anti-apoptotic protein Bcl-2 could be inhibited by high glucose induction, while the expression of anti-apoptotic protein Bcl-2 could be up-regulated by luteolin (fig. 5D). These results indicated that luteolin had the effect of inhibiting apoptosis. Western blot results showed that the expression of p-VEGFR2 was upregulated by high glucose, while luteolin inhibited the expression of p-VEGFR2 (fig. 5E). Further detection 

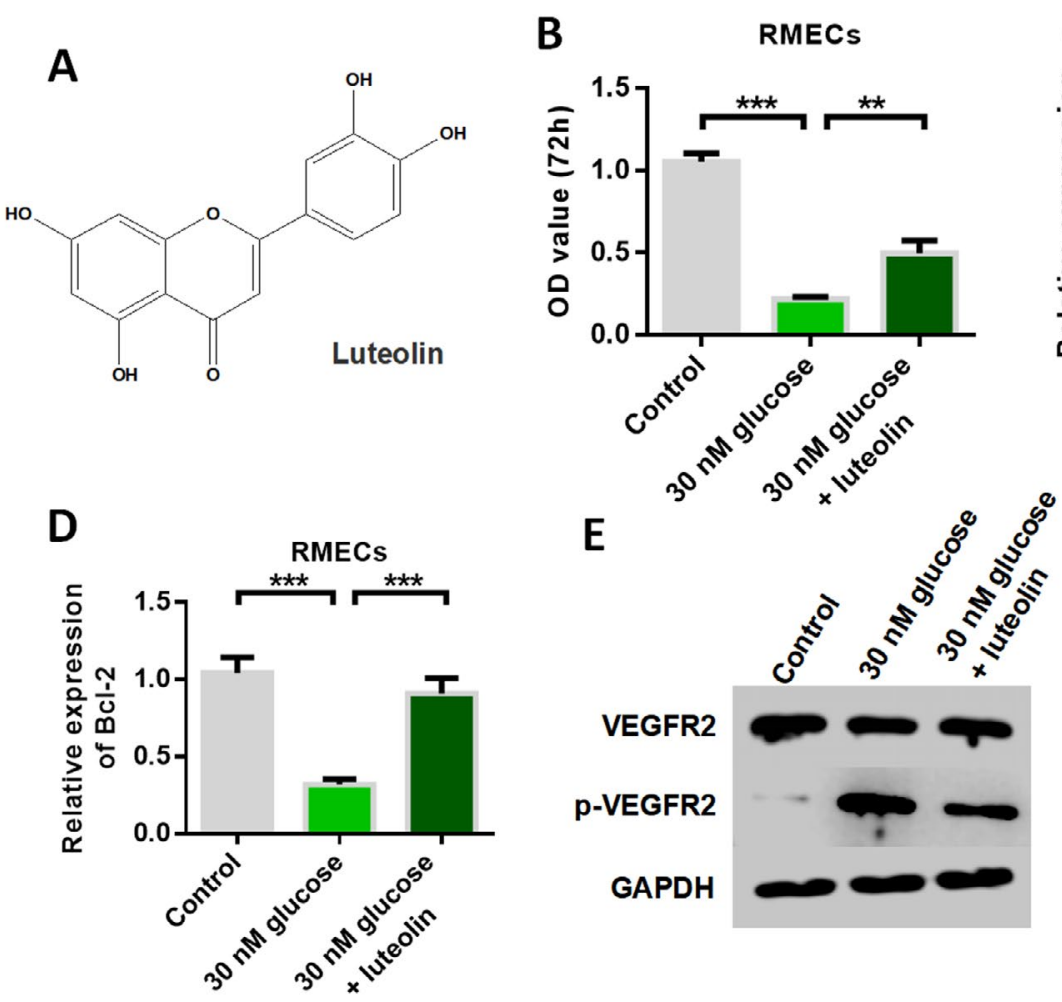

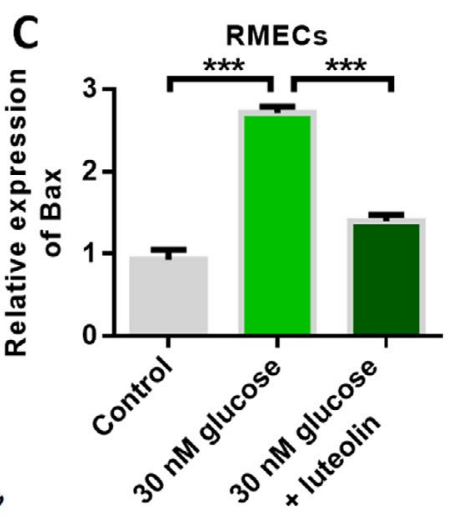

Fig. 5: Effect of luteolin on high glucose-treated RMECs Luteolin (A) treatment reversed the effects of high glucose treatment on B. RMECs C. Bax D. Bcl-2 and E. VEGFR2 and p-VEGFR2 in retinal microvascular endothelial cells (RMECs)

results of inflammatory cytokines showed that the expression levels of IL-6, TNF $\alpha$, LDH, IL- $1 \beta$ and MCP1 could be up-regulated by high glucose induction, and glucose-induced increased release of inflammatory cytokines. Then, the content of inflammatory cytokines significantly decreased after luteolin treatment, indicating that the mailuoning preferred to suppress the high glucose-induced cell inflammation (fig. 6).

Exposure to high glucose could result in diabetes-related microvascular and macrovascular complications ${ }^{[17,18]}$. Furthermore, it is well-documented that high glucose treatment could contribute to diabetic microvascular lesion. DR is the manifestation of diabetic microvascular lesions in the unique environment of the fundus, mainly caused by chronic high glucose ${ }^{[19,20]}$. High glucose may result in tissue ischemic hypoxia, which was related to a series of pathological changes, including leakage of retinal microvessels, retinal tissue edema, vitreous haemorrhage, tractional retinal detachment and blindness. The early changes of DR were presented as retinal injury, thickening of capillary basement membrane, loss of peripheral cells and abnormal apoptosis of RMECs. Recent studies showed that inhibition of apoptosis of RMECs could protect against $\mathrm{DR}^{[5,21-23]}$. The present study aim was to investigate the role of mailuoning and luteolin in preventing high glucose-induced RMECs apoptosis in DR.

Long-term chronic microvascular damage is the typical characteristic of the progress of DR. Meanwhile, high glucose and hypoxia are the two common and leading causes of diabetic vascular injury. Hence, experimental models of DR were widely constructed under high glucose or hypoxic conditions. Accumulated evidence revealed that abnormal apoptosis and dysfunction of RMECs contribute to the initial etiology ofmicrovascular injury that leads to DR. In the present study, a model of high glucose-induced DR was constructed by exposing RMECs to high glucose concentration to explore the mechanisms of the effect of mailuoning on apoptosis of RMECs.

The hypotheses on how high glucose could cause microangiopathy included, increased polyol pathway, increased formation of glycosylation end products, activation of protein kinase $\mathrm{C}$ subtype, increased hexosamine pathway and increased production of mitochondrial reactive oxygen species. These pathways could induce oxidative stress, inflammation and vascular dysfunction, leading to increased vascular permeability, vascular occlusion and ischemia. At the same time, these reactions would lead to the upregulation 
A
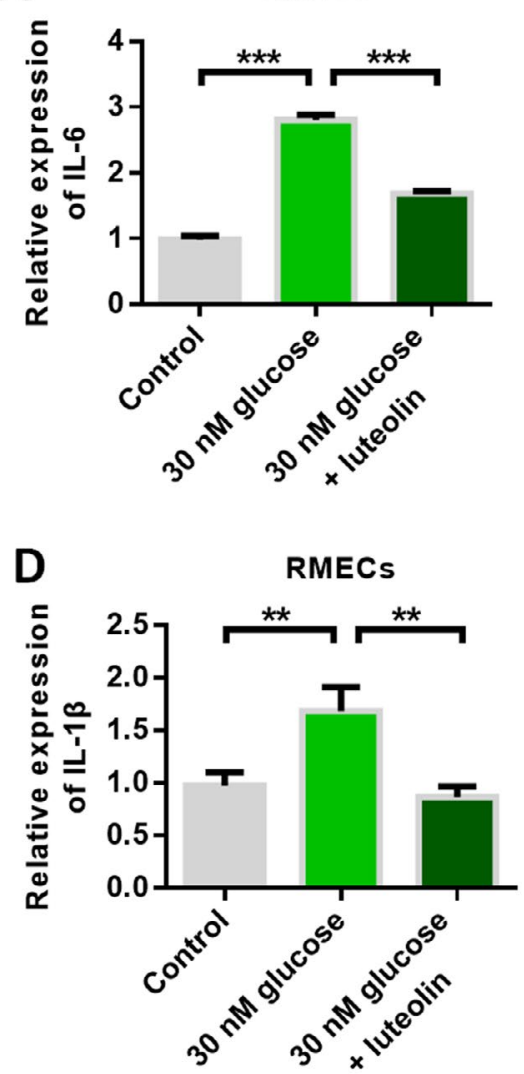

B

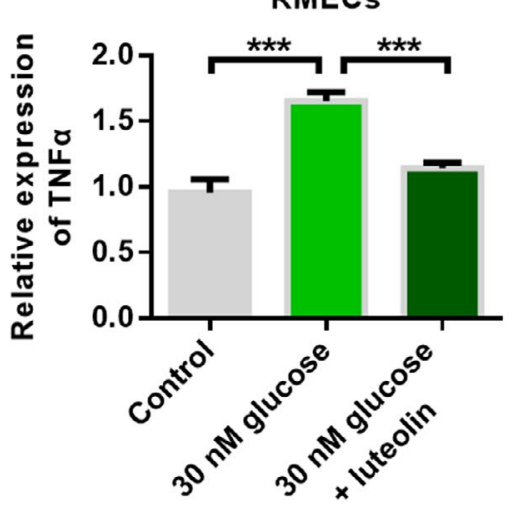

E

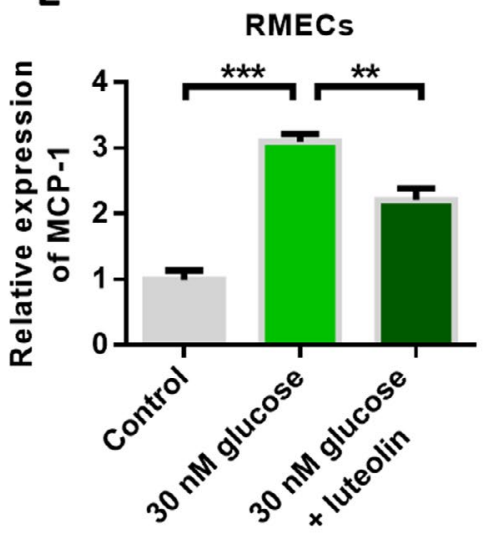

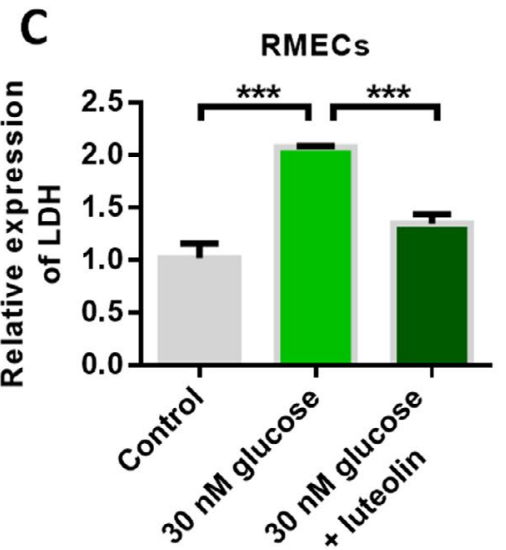

Fig. 6: Effect of high glucose level and luteolin on inflammatory factors in RMECs

High glucose level induced and treatment with luteolin inhibited the induction of A. IL-6, B. TNFa, C. LDH, D. IL-1ß and E. MCP-1 in retinal microvascular endothelial cells (RMECs)

of angiogenic factors and inflammatory factors, such as vascular endothelial growth factor (VEGF), insulin-like growth factor, angiopoietin-2 (Ang-2), interstitial-derived factor-1, fibroblasts growth factor-2, hepatocyte growth factor, tumor necrosis factor (TNF) and interleukin-6 (IL-6) ${ }^{[24,25]}$. This study found that high glucose concentration could inhibit retinal endothelial cell proliferation, promote the release of inflammatory factors and increase retinal endothelial cell apoptosis. At the same time, high glucose level could upregulate the expression of p-VEGFR2 ${ }^{[26,27]}$. Mailuoning and luteolin inhibited high glucose-induced effects such as, RMEC apoptosis, production of inflammatory factors and at the same time phosphorylation of p-VEGFR2, to play a role in the treatment of DR.

As a Chinese traditional medicine, mailuoning has been widely studied in various realms. For instance, mailuoning is used to treat acute ischaemic stroke, ischaemic injury, protect the heart and brain issues ${ }^{[28,29]}$. Previous studies showed that mailuoning injection exerted antiplatelet 3 and antithrombotic effects. Our previous study presented that mailuoning is capable of inhibiting high-glucose-triggered HUVEC apoptosis, indicating mailuoning is involved in the occurrence and development of diabetic mellitus ${ }^{[30]}$. In this study, it was found that $200 \mu \mathrm{g} / \mathrm{ml}$ mailuoning is the highest concentration without any cytotoxic effects. Meanwhile, after high glucose treatment, RMEC viability was significantly decreased, the reduction could be partially reversed by adding mailuoning in a concentrationdependent manner and $100 \mu \mathrm{g} / \mathrm{ml}$ mailuoning was the optimum concentration on RMECs. Furthermore, mailuoning could counteract the inhibition of RMEC apoptosis triggered by high glucose inDR. Luteolin could also protect against RMECs damage caused by high glucose by inhibiting VEGFR and its phosphorylation level ${ }^{[31,32]}$. This study found that compared to the high glucose group, the proliferation ability of RMECs in the high glucose+luteolin group was significantly increased, the apoptosis was significantly reduced, the level of cellular inflammatory factors was significantly reduced, and p-VEGFR2 expression was significantly reduced. For the first time, it was confirmed that luteolin could protect high glucose-induced RMECs, which may be related to the inhibition of p-VEGFR2 activation. 
The results of this study indicated that mailuoning and luteolin could attenuate the apoptosis induced by high glucose. Hence, mailuoning formula may be a novel and promising medication to treat DR in future.

\section{Acknowledgements:}

This work was supported by the Science and Technology Development Plan Project of Nanjing (No. 201715063).

\section{Conflict of interest:}

All authors report no conflicts of interest in this work.

\section{REFERENCES}

1. Fante RJ, Gardner TW, Sundstrom JM. Current and future management of diabetic retinopathy: a personalized evidencebased approach. Diabetes Manag 2013;3:481-94.

2. Li YY, Yang XF, Gu H, Snellingen T, Liu XP, Liu NP. The relationship between insulin resistance/ $\beta$-cell dysfunction and diabetic retinopathy in Chinese patients with type 2 diabetes mellitus: the Desheng Diabetic Eye Study. Int J Ophthalmol 2018;11:493-500.

3. Chen SC, Hsiao PJ, Huang JC, Lin KD, Hsu WH, Lee YL, et al. Abnormally Low or High Ankle-Brachial Index Is Associated With the Development of Diabetic Retinopathy in Type 2 Diabetes Mellitus. Sci Rep 2018; 8:1-7.

4. Lawrenson JG, Graham-Rowe E, Lorencatto F, Burr J, Grimshaw JM. Interventions to increase attendance for diabetic retinopathy screening. Acta Ophthalmologica 2018;1:CD012054.

5. Karim H, Asif B, Jake P, Swaminathan R. Concentration of Circulating Rhodopsin mRNA in Diabetic Retinopathy. Clin Chem 2020; 50:2152-5

6. Hacibekiroglu S, Michael I, Mitrousis N, Yang H, Nagy. Combined cell and gene therapy towards the treatment of age-related macular degeneration and diabetic retinopathy. Cytotherapy 2018;20:S19.

7. Li WL, Wang WT, Cao XP, Zhang ZH. Determination of chlorogenic acid in mailuoning injection by HPLC. J Chin Pharm Sci 2003;38:210-1

8. Xie Y, Chen L, Ding X, Jiang J, Zhang Y, Chen M. Effect of different doses of mailuoning injection on rats after cerebral ischemia reperfusion injury. Prog Mod Biomed 2010;10:282630 .

9. Chen A. Comparison of clinical effect of puerarine, ligustrazin and mailuoning in treating labour angina pectorisin treating labour angina pectoris. Chin J Integr Med 2000;6:144-5.

10. Zhang J, Wen-Zheng JU, Chen M, Liu SJ, Tan HS. Pharmacokinetic studies of mailuoning injection in rats. Chin Pharm Bulletin 2008;24:558-9.

11. Luan F, Yin HY, Dong PY. Observation on Treatment of mailuoning Injection for 46 Pregnancy Induced Hypertension Patients. Chin J Integr Med 1995;15:153-5.

12. Guo Q, Wu X, Wu N, Di L. Quantitative analysis of gigantol in mailuoning injection. Chinese J Pharm Anal 2011;31:236-9.

13. Peters N, Frost J, Long S. A plant flavone, luteolin, induces expression of Rhizobium meliloti nodulation genes. Science 1986;233:977-980.

14. Kimata M, Shichijo M, Miura T, Serizawa I, Inagaki N,
Nagai H. Effects of luteolin, quercetin and baicalein on immunoglobulin E-mediated mediator release from human cultured mast cells. Clin Exp Allergy 2000;30:501-8.

15. Kimata M, Inagaki a, Naoki, Nagai H. Effects of Luteolin and Other Flavonoids on IgE-Mediated Allergic Reactions. Planta Medica 2000;66:25-9.

16. Fan HW, Zhu Q, Hong M, Li YU. Analysis of active components in mailuoning injection by platelet membrane immobilized chromatography. J Chin Pharm Sci 2006;41:63-6.

17. Fiori A, Terlizzi V, Kremer H, Gebauer J, Bieback K. Mesenchymal stromal/stem cells as potential therapy in diabetic retinopathy. Immunobiology 2018; 223:729-43

18. Bain S. Semaglutide, reduction in glycated haemoglobin and the risk of diabetic retinopathy. Diabetes Obes Metab 2018;20:889-97.

19. Zhu XR, Yang Fy, Lu J, Zhang H, Yang JK. Plasma metabolomic profiling of proliferative diabetic retinopathy. Nutr Metab 2019;16:37.

20. Zeng Y, Cao D, Yang D, Zhuang X, Yu H, Hu Y, et al. Screening for diabetic retinopathy in diabetic patients with a mydriasisfree, full-field flicker electroretinogram recording device. Documenta ophthalmologica. Doc Ophthalmol 2020;140:211220.

21. Sever O, Horozoglu F. Bariatric surgery might aggravate proliferative diabetic retinopathy. Acta Ophthalmologica 2020.

22. Kohl K, Kolibabka M, Friedrichs P, Hammes HP. H3K36me3 associated pericyte loss in early diabetic retinopathy. Diabetol Stoffwechs 2018;13:131.

23. Nishimura A, Kasai T, Kikuno S, Nagasawa K, Okubo M, Narui K, et al. Apnea Hypopnea Index During Rapid Eye Movement Sleep With Diabetic Retinopathy in Patients With Type 2 Diabetes. J Clin Endocrinol Metab 2019;104:2075-82.

24. Ogura S, Kurata K, Hattori Y, Takase H, Ishiguro-Oonuma $\mathrm{T}$, Hwang Y, et al. Sustained inflammation after pericyte depletion induces irreversible blood-retina barrier breakdown. Jci Insight 2017;2.

25. Muthusamy A, Lin CM, Lindner H, Shanmugam S, Abcouwer $\mathrm{S}$, Antonetti D. Early Ischemia-reperfusion Injury Induces Retinal Vascular Permeability in a VEGF Receptor 2 Dependent Manner Followed by Occludin Phosphorylation and Ubiquitination. Invest Ophthalmol Vis Sci 2013;54-2474.

26. Liu H, Zhang W, Xu Z, Caldwell RW, Caldwell RB, Brooks SE. (Hyperoxia Causes Regression of Vitreous Neovascularization by Downregulating VEGF/VEGFR2 Pathway. Invest Ophthalmol Vis Sci 2013;54:918-31.

27. Mondal LK, Choudhury S, Paine SK, Sen A, Bhaduri G, Bhattacharya B. Anaerobic glycosis is the centre of different biochemical disarrays associated with the onset of diabetic retinopathy. JEMDS 2013;2:2320-9.

28. Zhi MX, Zhang GB, Hou JC, Yang YH, Wan ZZ. Peridural injection of mailuoning compound liquor for treatment of prolapse of lumbar intervertebral disc in 100 Cases. J Tradit Chin Med 2009;29:6-8.

29. Yu L, Li Y, Fan H, Duan J, Zhu Q, Li S. Analysis of marker compounds with anti-platelet aggregation effects in mailuoning injection using platelet binding assay combined with HPLC-DAD-ESI-MS and solid-phase extraction technique. Phytochem Anal 2011;22:87-93.

30. Pang XB, Xie XM, Wang HY, Wang BQ. Protective effect of mailuoning injection on cerebral ischemia/reperfusion injury in rats and its mechanism. China Journal of Chinese Materia Medica 2004;39(4):721-725. 
31. Xagorari A, Papapetropoulos A, Mauromatis A, Economou M, Roussos C. Luteolin inhibits an endotoxin-simulated phosphorylation cascade and proinflammatory cytokine production in macrophages. J Pharmacol Exp Ther 52001;296:181-7.

32. Shimoi K, Okada H, Furugori M, Goda T, Takase S, Suzuki $\mathrm{M}$, et al. Intestinal absorption of luteolin and luteolin 7-O- $\beta$ glucoside in rats and humans. Febs Letters 1998;438:220.
This is an open access article distributed under the terms of the Creative Commons Attribution-NonCommercial-ShareAlike 3.0 License, which allows others to remix, tweak, and build upon the work non-commercially, as long as the author is credited and the new creations are licensed under the identical terms

This article was originally published in a special issue, "Biomedical Research in Healthcare Setting" Indian J Pharm Sci 2020:82(2)Spl issue5;6-15 